\title{
Perspektiven eines Europäischen Arbeitsmarktes
}

\author{
Werner Tegtmeier
}

A. Einleitung

B. Ausgewählte, das Arbeitskräftepotential beeinflussende Faktoren 128

$\begin{array}{ll}\text { I. Demographie } & 128\end{array}$

II. Die Komponente der Altersgrenzen $\quad 129$

III. Qualitative Aspekte 131

C. Ausgewählte, die Nachfrage nach Arbeitskräften berührende Aspekte 132

I. Die Lissabon-Strategie - zwischen Anspruch, Wirklichkeit und Notwendigkeiten

II. Arbeitsmarktstabilisatoren 133

III. Arbeitnehmerfreizügigkeit - Wanderungen 134

\section{A. Einleitung}

Nachdenken über mittel- und längerfristige Perspektiven eines Europäischen Arbeitsmarktes heißt, relevante Aspekte zur Entwicklung des Arbeitskräftepotentials und der Nutzung dieses Potentials, der Nachfrage nach Arbeitskräften, zu benennen. Zum 1. Mai 2011 ist die volle Freizügigkeit der Arbeitnehmer auch für die im Jahre 2004 hinzugekommenen Mitglieder der EU wirksam geworden. Und zugleich sind dunkle Wolken für Beschäftigung und Arbeitsmarkt im Gefolge der Staatsschulden- und Finanzkrise aufgezogen, die nicht nur die erzielten beschäftigungspolitischen Fortschritte in Frage stellen, sondern auf Jahre die makroökonomischen Möglichkeiten für mehr Beschäftigung beeinträchtigen werden, und sie werden die bisherige Statik der EU selbst berühren und verändern ${ }^{1}$.

Die von den realwirtschaftlichen und finanziellen Auswirkungen des Zusammenbruchs von Lehman Brothers und ihrer eigenen Staatsverschuldung besonders betroffe-

1 Weder di Mauro, Mitglied des Sachverständigenrates der Bundesregierung zur Begutachtung der gesamtwirtschaftlichen Entwicklung vermutet sogar: „Der Zerfall Europas hat längst begonnen“ (Süddeutsche Zeitung v. 14.11.2011, S. 16) und Bundeskanzlerin Merkel spricht in diesem Kontext von einer „Zeit epochaler Veränderungen in der Welt“ (SZ v. 15.11.11, S. 1), „Wir sind in einer wirklichen systemischen Krise“, Kommissionspräsident Barroso vor dem EU-Parlament in Straßburg (SZ v. 17.11.2011, S. 1). 
nen Mitgliedstaaten der EU zeigen bereits im Jahresvergleich September 2009 zu September 2010 einen deutlichen Anstieg der harmonisierten Arbeitslosenquoten, z.B. Irland von $12,5 \%$ auf $13,9 \%$, Spanien von $19,0 \%$ auf $20,6 \%$, Portugal von $10,0 \%$ auf $11,2 \%$, Griechenland von 9,7\% auf 12,9\% ${ }^{2}$. Die aktuellen Herausforderungen im Europäischen Arbeitsmarkt sind bereits hoch, und sie werden noch zunehmen.

\section{B. Ausgewählte, das Arbeitskräftepotential beeinflussende Faktoren}

\section{Demographie}

Seit den 60er Jahren des vorherigen Jahrhunderts hat sich in Europa ein tiefgreifender Wandel in der demographischen Entwicklung vollzogen. Während im Jahr 2011 die Weltbevölkerung die 7-Milliarden-Grenze überschritten hat, wird nach Modellrechnungen der UN die Bevölkerung der EU-27 im Zeitraum zwischen 2005/10 und 2045/50 „,von 490 Millionen auf 455 Millionen“ schrumpfen. Ursächlich hierfür ist die andauernd niedrige Fertilität mit einer Nettoreproduktionsrate unter $1^{3}$. Dieses veränderte generative Verhalten der Bevölkerung ist zunächst in Deutschland besonders deutlich ausgeprägt, manifestiert sich dann - zeitversetzt - mit ähnlicher Tendenz in den südlichen und östlichen Staaten der Union. Hierzu einige Beispiele zur Entwicklung der NRP für den Zeitraum 2005 - 2010 (1995 - 2000): Deutschland 0,59 (0,66), Frankreich $0,91(0,87)$, Italien $0,66(0,63)$, Polen 0,59 $(1,03)^{4}$.

$\mathrm{Zu}$ diesem Sachverhalt tritt hinzu, dass die Lebenserwartung der Menschen seit gut 2 Jahrzehnten eine - noch andauernd - steigende Tendenz aufweist. Zur Illustration: In Deutschland betrug nach der Sterbetafel 1991/93 die Lebenserwartung eines neugeborenen Knaben 72 Jahre und für neugeborene Mädchen 79 Jahre. Gegenwärtig beträgt sie für neugeborene Jungen 77 Jahre, für neugeborene Mädchen 82 Jahre. Und auch die spätere Lebenserwartung ist im gleichen Zeitraum deutlich angestiegen - für Frauen im 60jährigen Alter von 22 auf 25 Jahre und für die 60jährigen Männer von 18 auf 21 Jah$\mathrm{re}^{5}$.

Die ausgewählten Fakten und beschriebenen Trends belegen: Die Bevölkerung wird im Zeitablauf abnehmen, und sie wird sich altersstrukturell nachhaltig anders zusam-

2 Stand: März 2011, Bundesministerium für Arbeit und Soziales (Hrsg.), Eurostat in Sozialkompass für Europa, 2011, S. 124.

3 Bundesinstitut für Bevölkerungsforschung, Bevölkerung. Daten, Fakten, Trends zum demographischen Wandel in Deutschland, 2008, S. 31. Die NRP = 1 besagt, dass die Frauengeneration im gebärfähigen Alter genauso viele Mädchen zur Welt bringt wie sie selbst zahlenmäßig repräsentiert. In diesem Fall bleibt die Bevölkerung quantitativ stabil.

4 Statistisches Bundesamt, Statisches Jahrbuch 2008 für die Bundesrepublik Deutschland, 2008, S. 677.

5 Bundesinstitut für Bevölkerungsforschung, Bevölkerung (Fußn. 3), S. 46 f. 
mensetzen. Dieser Trend gilt, z.T. zeitversetzt, im Kern für die gesamte EU-27. Für das Arbeitskräftepotential hat das gravierende Auswirkungen, ebenso für die Finanzierung und Leistungsfähigkeit der Systeme sozialer Sicherung in den Mitgliedstaaten. Während die beschriebene demographische Entwicklung für die nächsten Jahrzehnte als nachhaltig vorgegeben und auch durch Wanderungen als nicht mehr signifikant veränderbar zu betrachten ist, stehen die zur Bewältigung dieser Herausforderungen notwendigen auch arbeitsmarktrelevanten - strukturellen Reformen vielfach noch aus bzw. erst am Anfang.

\section{Die Komponente der Altersgrenzen}

Das Arbeitskräftepotential wird im Kontext der demographischen Entwicklung von drei Komponenten besonders beeinflusst: Alter beim Eintritt ins Erwerbsleben, Erwerbsbeteiligung (vgl. hierzu auch Anlagen 3 - 5), Alter beim Ausscheiden aus dem Erwerbsleben. Hier soll letzterer Aspekt näher beleuchtet werden.

Zuvor einige Fakten und abgeleitete Daten für die Besetzung der relevanten Altersgruppen (unter 20 Jahre, 20 bis unter 65 Jahre, 65 Jahre und älter) im Zeitraum 20102050 für Deutschland auf der Basis der 11. koordinierten Bevölkerungsvorausberechnung6:

\begin{tabular}{|c|c|c|c|}
\hline Jahr & unter 20 J. & 20 bis unter 65 J. & 65 J. und älter \\
\hline 2005 & 20,0 & 60,8 & 19,3 \\
\hline 2010 & 18,3 & 61,1 & 20,5 \\
\hline 2020 & 16,9 & 60,2 & 22,9 \\
\hline 2030 & 16,6 & 55,5 & 27,9 \\
\hline 2040 & 16,0 & 53,2 & 30,8 \\
\hline 2050 & 15,4 & 52,8 & 31,8 \\
\hline
\end{tabular}

Heute sind in Deutschland knapp 50 Mio. Personen im Alter von 20 bis unter 65 Jahren, 2 Jahrzehnte später werden es nach dieser Schätzung der Demographen nur noch knapp 44 Mio. Personen sein, und für 2050 wird die Zahl der Personen im Erwerbsalter zwischen 36 bzw. rund 39 Mio. Personen veranschlagt, also gut ein Fünftel weniger als derzeit.

Die Gesamtbevölkerung wird unter den gewählten Annahmen im gleichen Zeitraum von derzeit knapp 82 Millionen auf in 2050 zwischen 69 bzw. 74 Mio. Personen zurückgegangen sein.

Das Bemerkenswerte dabei ist: Wenn man Irland (NRP rund 1) und Luxemburg (Zuwanderung) außen vor lässt, dann werden die Arbeitsmärkte der EU-27 alle, wenn

6 Bundesinstitut für Bevölkerungsforschung, Bevölkerung (Fußn. 3), S. 16 f. 
auch mit unterschiedlichen Tempi, eine ähnliche Entwicklung erfahren. Beispiel Polen: Die stark gesunkene Fertilität wird dazu führen, dass Polen, das derzeit noch unter dem EU-27 Durchschnitt bei den „65-jährigen und älter“ liegt, bis 2050 deutlich über dem EU-27 Durchschnitt liegen wird7.

Die beschriebene Entwicklung hat tiefgreifende Auswirkungen auf nahezu alle Politikbereiche - auf die makroökonomischen Aggregate, auf die Bildungspolitik, auf die Finanzierbarkeit sozialer Sicherung. Und für das Beschäftigungssystem und die Arbeitsmärkte bedeutet dies:

- technischer Fortschritt und Wertschöpfungserstellung werden künftig mit einem altersstrukturell anderen, nämlich älter zusammengesetzten Erwerbspersonenpotential bewältigt werden müssen (Anlage 3);

- die Lebensarbeitszeit muss wieder ausgeweitet und bestehende Beschäftigungspotentiale dürfen nicht brach liegen gelassen werden;

- die Balance zwischen Finanzierbarkeit und Leistungsfähigkeit sozialer Sicherung, hier des Anteils der Wertschöpfung, der auf die nicht erwerbstätige Bevölkerung entfällt, muss neu justiert werden.

In Deutschland ist mit dem Rentenversicherungs-Altersgrenzenanpassungsgesetz eine Regelung wirksam geworden, die ab dem Jahr 2012 die Regelaltersgrenze stufenweise vom 65. auf das 67. Lebensjahr anhebt. Für die Versicherten der Geburtsjahrgänge 1947 - 1958 erfolgt diese Anhebung in 1-Monats-Schritten pro Geburtsjahrgang, für die Versicherten der Jahrgänge 1959 - 1964 mit 2 Monaten pro Geburtsjahrgang, so dass ab Geburtsjahrgang 1964 das Renteneintrittsalter bei 67 liegen wird ${ }^{8}$.

Zur Betrachtung dieser arbeitsmarktrelevanten Sachverhalte tritt hinzu, dass bei vorzeitiger Inanspruchnahme der Altersrente ein Abschlag von 0,3\% pro Monat wirksam wird, und dass die rentenpolitische Zielsetzung einen Paradigmenwechsel dergestalt erfahren hat, dass als Steuerungsgröße nicht mehr ein bestimmtes Rentenniveau, sondern eine Beitragsobergrenze handlungsleitend ist: Bis 2020 kein Überschreiten von 20\%, bis 2030 kein Überschreiten von 30\%. Durch die Einführung eines „Nachhaltigkeitsfaktors" ${ }^{\text {"9 }}$ und anderer, die jeweiligen Rentenanpassungen abflachender Komponenten in die Rentenformel wird das Rentenniveau heruntergefahren. Zielmarke: Bis 2020 sollen $46 \%$, bis $203043 \%$ des Rentenniveaus vor Steuern nicht unterschritten werden ${ }^{10}$ - mit dem Ergebnis, dass eine auskömmliche Alterssicherung aus der gesetzlichen Ren-

7 Bundesinstitut für Bevölkerungsforschung, Bevölkerung (Fußn. 3), S. 31.

8 Flecken, Sozialgesetzbuch - 6. Buch - Rentenversicherung, in: Bundesministerium für Arbeit und Soziales (Hrsg.) Übersicht über das Sozialrecht (Ausgabe 2011/2012), 8. Aufl. 2011, S. 333 f.

9 Der Nachhaltigkeitsfaktor spiegelt die Veränderung des Verhältnisses von Leistungsbeziehern und sozialversicherungspflichtig beschäftigten Personen im vorangegangenen Kalenderjahr wider. Die Regelung ist seit 2005 wirksam.

10 Flecken, SGB VI (Fußn. 8), S. 315. 
tenversicherung ohne komplementäre Vorsorge (betriebliche und individuelle Vorsorge) in der Regel nicht mehr erreichbar sein wird ${ }^{11}$.

Es ist evident, dass die Änderung dieser Rahmenbedingungen das bisherige Verhalten der Arbeitsmarktakteure verändern und entsprechende Auswirkungen auf das Arbeitskräftepotential haben wird. In der EU-27 ist der Prozess zur Anhebung der Regelaltersgrenze in vollem Gange: Deutschland wird die Anhebung auf das 67. Lebensjahr bis 2029 abgeschlossen haben; Dänemark bis 2027 und Dänemark hat darüber hinaus die Absicht, eine Koppelung mit der Entwicklung der Lebenserwartung zu verwirklichen; Ungarn wird die Regelaltersgrenze bis 2022 von 62 auf 65 Jahre angehoben haben; Rumänien erhöht bis 2014 bei den Männern (63 Jahre) und Frauen (58 Jahre) um jeweils 2 Jahre; Frankreich hat sie für „langjährig Versicherte auf 62 Jahre und für die restlichen Versicherten auf 67 Jahre erhöht. Großbritannien plant die Erhöhung auf 66 Jahre bis 2020." 12 Und Polen ${ }^{13}$ hat ebenfalls eine Anhebung der Altersgrenze auf das 67. Lebensjahr (bis 2027) angekündigt.

\section{Qualitative Aspekte}

Für die Nutzung des jeweiligen Arbeitskräftepotentials spielen qualitative Aspekte eine besondere Rolle: die schulische und berufliche Qualifikation, die Befähigung zum lebenslangen Lernen, um mit dem technischen Fortschritt mithalten zu können, die Flexibilität, um auch unter veränderten Rahmenbedingungen produktive Wertschöpfung zu erbringen. Das sind Anforderungen, die sich sowohl an das Bildungs- und Beschäftigungssystem und das Steuer- und Sozialsystem und die dort gesetzten Regelungsmechanismen als auch an die jeweiligen Individuen richten. Und hier fällt auf, dass einzelwirtschaftliche, individuelle und gesamtwirtschaftliche, gesamtgesellschaftliche Rationalität - nicht nur gelegentlich - in herber Weise auseinanderklaffen ${ }^{14}$. Und das Gleiche gilt auch für den Zeit- und Sachhorizont vieler Entscheidungsträger. Die jahrelang geübte Praxis der Frühverrentung ist dafür ein besonders anschauliches Beispiel. Es war eben „vermeintlich“ kostengünstiger, eine Beschäftigungsanpassung auf diese Weise, nämlich faktisch als Vertrag zu Lasten Dritter (des Steuer- bzw. Sozialversicherungssystems) $)^{15}$ abzuwickeln, als neue innovative und künftige Generationen nicht be-

11 Kritische Anmerkungen hierzu bei Schmähl, Warum ein Abschied von der ,,neuen deutschen Alterssicherungspolitik“ notwendig ist, in: ZeS (Zentrum für Sozialpolitik) - Arbeitspapier Nr. 01/2011, S. $11 \mathrm{ff}$.

12 Bundesministerium für Arbeit und Soziales (Hrsg.), Sozialkompass Europa. Soziale Sicherheit im Vergleich, 2011, S. 140.

13 Regierungserklärung Premier Tusk v. 18.11.2011.

14 Tegtmeier, Arbeitsmarktpolitik - Anspruch und Wirklichkeit von Arbeitsmarktreformen, ZeS - Arbeitspapier Nr. 5/2007, S. $10 \mathrm{f}$.

15 Börsch-Supan untersucht dies am Beispiel der Erwerbsminderungsrenten in einer Reihe von Wohlfahrtsstaaten (u.a. auch Polen und Deutschland) und kommt zu dem Ergebnis, dass insbes. „Groß- 
lastende Anpassungsformen ${ }^{16}$ zu wählen: In der EU-27 beendeten die Arbeitnehmer ihre Erwerbstätigkeit durchschnittlich zwischen 58,7 Jahren (Slowakei) und 64,1 Jahren (Irland), für Deutschland betrug der Durchschnittswert 61,7 Jahre. ${ }^{17}$

\section{Ausgewählte, die Nachfrage nach Arbeitskräften berührende Aspekte}

\section{Die Lissabon-Strategie - zwischen Anspruch, Wirklichkeit und Notwendigkeiten}

Die Beschlüsse der Staats- und Regierungschefs im Jahr 2000 in Lissabon waren noch von der Vision geprägt, die EU binnen eines Jahrzehnts zum wettbewerbsfähigsten und dynamischsten Wirtschaftsraum der Welt werden zu lassen und als wichtige Pfeiler Innovation, Wissensgesellschaft und soziale Kohäsion voranzubringen. ${ }^{18}$ Die Realität von Staatsschulden-, Finanz- und Bankenkrise haben dagegen - besonders in der PIIGS Gruppe $^{19}$ - zu schwerwiegenden Beeinträchtigungen von Wachstum und Beschäftigung geführt, und der Instrumenteneinsatz trägt in vielen Bereichen sogar noch prozyklische, nämlich krisenverschärfende Züge. Mittlerweile sind in der genannten Ländergruppe alle Regierungen umgebildet oder abgewählt worden - zuletzt in Spanien und in Italien.

Allein in Spanien sind z.Z. über ein Fünftel der Menschen arbeitslos; bei den Jugendlichen ist nahezu jeder zweite, bei den Universitätsabsolventen jeder fünfte von Arbeitslosigkeit betroffen.

Deutschland befindet sich dagegen noch auf einem, wenn auch abgeschwächten Wachstumspfad, und in zahlreichen Bereichen wird aktueller und künftig tendenziell noch zunehmender Mangel an Fachkräften beklagt.

In Europa fehlt es an einer Perspektive für zukunftsträchtige Investitionen. Dabei gäbe es riesige Bereiche, von der Energieinfrastruktur (Beispiel: DESERTEC ${ }^{20}$ ) bis zu den großen Verkehrs- und Transportmagistralen, die Zukunft mit Beschäftigung verbinden würden und die die prozyklischen Wirkungen von allein auf Sparen und Konsolidieren gerichtetem Handeln durchbrechen würden.

zügigkeit und Zugänglichkeit“" der institutionellen Ausgestaltung dieser Systeme rd. 75\% der internationalen Querschnittsvariation erklären. Vgl. Börsch-Supan, Health and disability insurance, Zeitschrift für ArbeitsmarktForschung, Volume 44, Number 4, 2011, S. 359 ff.

16 Zum Beispiel durch ein intelligentes Zeitmanagement (Arbeitszeitkonten).

17 Bundesministerium für Arbeit und Soziales (Hrsg.), Sozialkompass, (Fußn. 12), S. 141.

18 Ebd., S. $31 \mathrm{f}$.

19 PIIGS - Portugal, Irland, Italien, Griechenland, Spanien.

20 DESERTEC ist eine Initiative zur Nutzung der solaren und solarthermischen Energiegewinnung in den Mittelmeer-Anrainerstaaten, insbes. Nordafrika. 


\section{Arbeitsmarktstabilisatoren}

Deutschland verfügt aus seiner jüngeren Geschichte über empirische Erfahrungen im Einsatz von Instrumenten der Arbeitsmarktpolitik bei gravierenden wirtschaftsstrukturellen Umbrüchen und auch bei konjunkturellen Einbrüchen.

Im Zuge der deutschen Vereinigung und des Zusammenbruchs der DDR-Wirtschaft wurde mit dem sogenannten strukturellen Kurzarbeitergeld ein Instrument eingesetzt, das die schlagartige Freisetzung nicht mehr benötigter Arbeitnehmer verhinderte und zugleich die bestehende Organisationsstruktur nutzte, um die technische Abwicklung der Leistung zu bewerkstelligen und - wo möglich - mit Qualifizierungsmaßnahmen durch geeignete Träger zu verbinden: Im Ergebnis also ein intelligenter Einsatz von ohnehin notwendigen Transferzahlungen mit investiven, nämlich qualifizierenden Elementen. In der Spitze und für einen kurzen Zeitraum umfasste dies rund ein Fünftel des seinerzeitigen Erwerbspersonenpotentials der DDR. Die seinerzeitige Befürchtung der Konservierung überholter, nicht wettbewerbsfähiger Strukturen hat sich nicht bestätigt, wohl aber dürften schwerwiegende soziale und gesellschaftliche Konflikte vermieden worden sein.

Auch der in der Folge des Zusammenbruchs von Lehman Brothers und der Bankenkrise in Deutschland erfolgte spürbare Wachstumseinbruch 2008/2009 wurde mit dem Instrument der Kurzarbeit arbeitsmarktwirksam entschärft und abgefangen. Hierzu wurde die Bezugsdauer von Kurzarbeitergeld mehrfach verlängert - in der Spitze bis auf 24 Monate, die Arbeitgeber wurden von den von ihnen zu tragenden Sozialversicherungsbeiträgen zur Hälfte entlastet, bei Qualifizierungsmaßnahmen während der Kurzarbeitsphase erfolgte sogar volle Erstattung durch die Bundesagentur für Arbeit. In der Spitze wurde diese Regelung von rund 1,4 Mio. Personen in Anspruch genommen. ${ }^{21}$ Zusammen mit unternehmensspezifischen Arbeitszeitregimes (Ansammlung von Überstunden auf Arbeitszeitkonten und Abbau in Phasen der Unterbeschäftigung) konnten die Stammbelegschaften relativ stabil gehalten und mit Anziehen der Konjunktur auch wieder unmittelbar produktiv eingesetzt werden.

Die Schattenseite dieses „Erfolges“ soll nicht verschwiegen werden: Vom Beschäftigungsabbau hart betroffen waren vor allem sog. Leiharbeitnehmer und Arbeitnehmer in befristeten Beschäftigungsverhältnissen.

21 Vergleich hierzu Bundesministerium für Arbeit und Soziales (Hrsg.), Sozialbericht 2009, S. 29 f. 


\section{Arbeitnehmerfreizügigkeit - Wanderungen}

Das Jahr 2011 bedeutet für die Arbeitnehmer der EU-27 (mit Ausnahme von Bulgarien und Rumänien, bei denen noch Übergangsfristen ${ }^{22}$ bis 2013 greifen) die Herstellung der vollen Freizügigkeit auf den Arbeitsmärkten. Bilaterale Abkommen, etwa Werksvertrags- oder Saisonarbeitnehmer - Vereinbarungen sind dadurch entbehrlich geworden. Die Koordinierung der Systeme der sozialen Sicherheit, Regelungen über die soziale Sicherung und über die Erbringung von Leistungen bei grenzüberschreitender Tätigkeit, Anrechnungsbestimmungen etc. sind in den einschlägigen Verordnungen ${ }^{23}$ für die EU und den EWR normiert und erlauben qualifizierte Information und Beratung derjenigen, die von der Freizügigkeit Gebrauch machen wollen. Ein Gesetz zur Verbesserung der Feststellung und Anerkennung im Ausland erworbener Bildungsabschüsse wird im Frühjahr 2012 in Deutschland wirksam werden. ${ }^{24}$ D.h., viele Barrieren der grenzüberschreitenden Mobilität von Arbeitskräften, die in früheren Forschungen noch zu beachten waren ${ }^{25}$, haben an Gewicht verloren, spielen in der Praxis aber gleichwohl noch eine erhebliche Rolle.

Es bedarf keiner Prophetie, dass angesichts der unterschiedlichen Beschäftigungssituation, des zum Teil extremen Anstiegs der Arbeitslosigkeit in einigen Ländern der EU-27, der Wanderungsdruck wieder zunehmen dürfte. Zusammen mit Armutswanderungen (aus Nordafrika in die Mittelmeeranrainerstaaten, Ostgrenzen der EU-27) und den Implikationen für die besonders betroffenen Länder kann daraus eine sehr brisante Lage erwachsen.

Freizügigkeit für die Arbeitnehmer ist ein hohes Gut, Ausgleich von Arbeitsmärkten ein sinnvoller ökonomischer Vorgang. Wenn dies allerdings im Ergebnis in der langfristigen Perspektive lediglich zur „passiven Sanierung“ ganzer Regionen führt, stellen sich durchaus einige Fragen. Eine Erkenntnis hieraus könnte sein, dass die durch Freizügigkeit verbesserte und gewollte Ausgleichsfunktion von Arbeitsmärkten eine gestaltende Struktur-, Wirtschafts-, Sozial- und Finanzpolitik eben nicht ersetzen kann. Am Beispiel der empirischen Befunde im Zuge der deutschen Vereinigung können Licht- und Schattenseiten von aktivem oder unterlassenem Handeln ja durchaus besichtigt werden. $\mathrm{Ob}$

22 Auch hier gelten die im Juni 2001 in Göteborg vereinbarten Übergangsregelungen: 2-3-2. D.h., jedes Mitgliedsland entscheidet individuell über die Inanspruchnahme der Übergangsfrist. In der ersten Stufe ohne Begründung, in der zweiten Stufe mit Begründung, in der 3. Stufe mit qualifizierter Begründung.

23 Kalmund, Internationale Sicherung, in: Bundesministerium für Arbeit und Soziales (Hrsg.), Übersicht über das Sozialrecht, 8. Aufl. 2011, S. 1089 f.

24 In der Bildungspolitik wird zurzeit (zusammen mit Universitäten und Tarifpartnern) an einem „Europäischen Qualifikationsrahmen“ gearbeitet, in dem einmal alle Bildungsabschlüsse in 8 Stufen eingeordnet werden sollen. Vgl. SZ v. 22.11.2011, S. 26.

25 Hönekopp, Arbeitsmarktperspektiven in der erweiterten Europäischen Union, in: Der Bürger im Staat H.1 2004, S. 34 ff.; auch neuere Untersuchungen zeigen, dass die Potenziale der Freizügigkeit nur begrenzt genutzt werden. Vgl. hierzu auch IAB-Kurzbericht 24/2011. 
daraus allerdings hinreichende Lehren - z.B. auch für den „Bau des Europäischen Hauses“ - gezogen worden sind, darf füglich bezweifelt werden. Die derzeitige Fokussierung der Diskussion Europäischer Perspektiven auf eine „Fiskalunion“ ist jedenfalls eine unangemessene Verkürzung der eigentlichen Gestaltungsaufgabe. 
Anlage 1:

Harmonisierte Arbeitslosenquote Sept. 2010 (Sept. 2009*) - Insgesamt in $\%$ (saisonbereinigt)

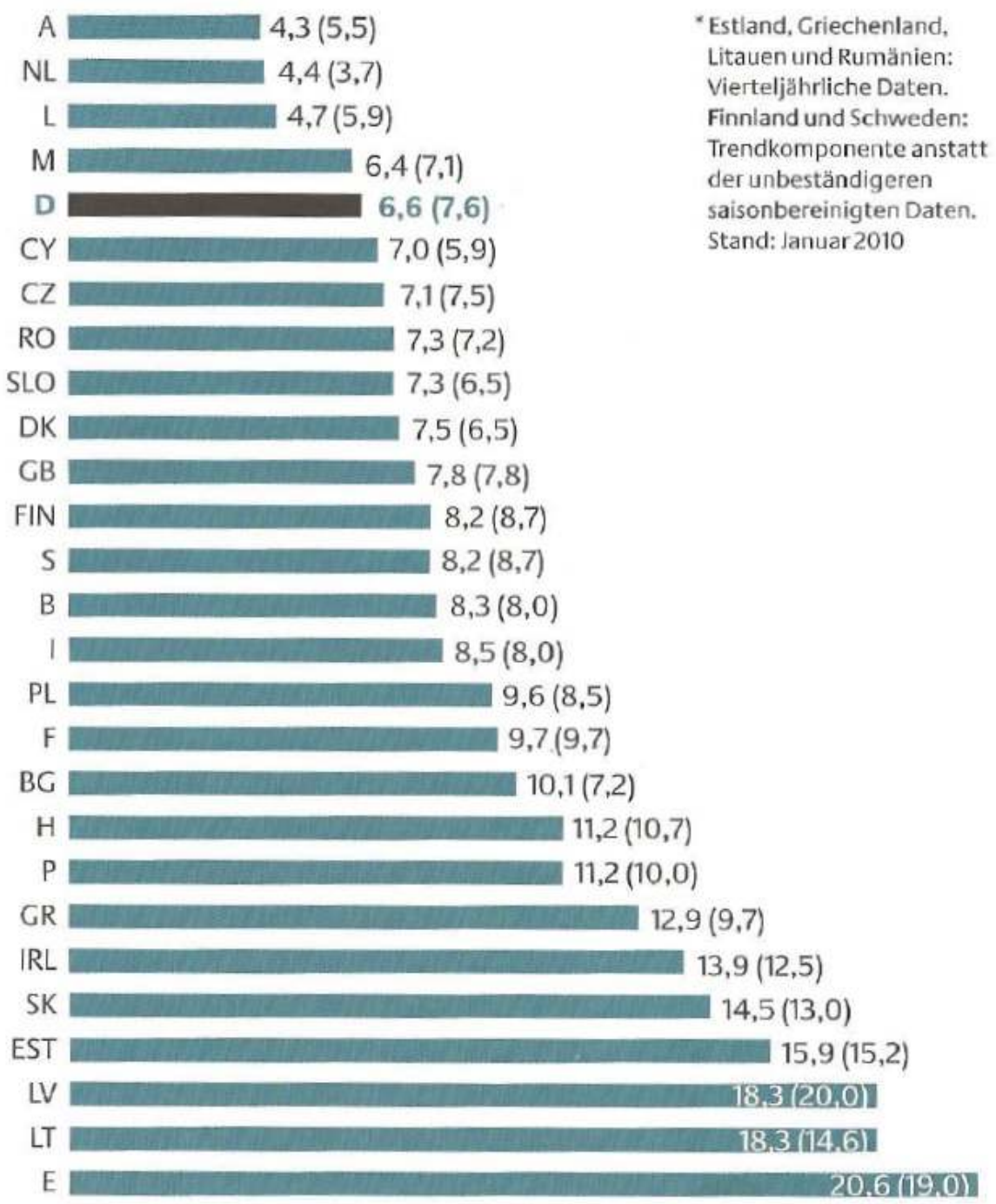

Stand: März 2011

Quelle: Eurostat 9. 
Anlage 2:

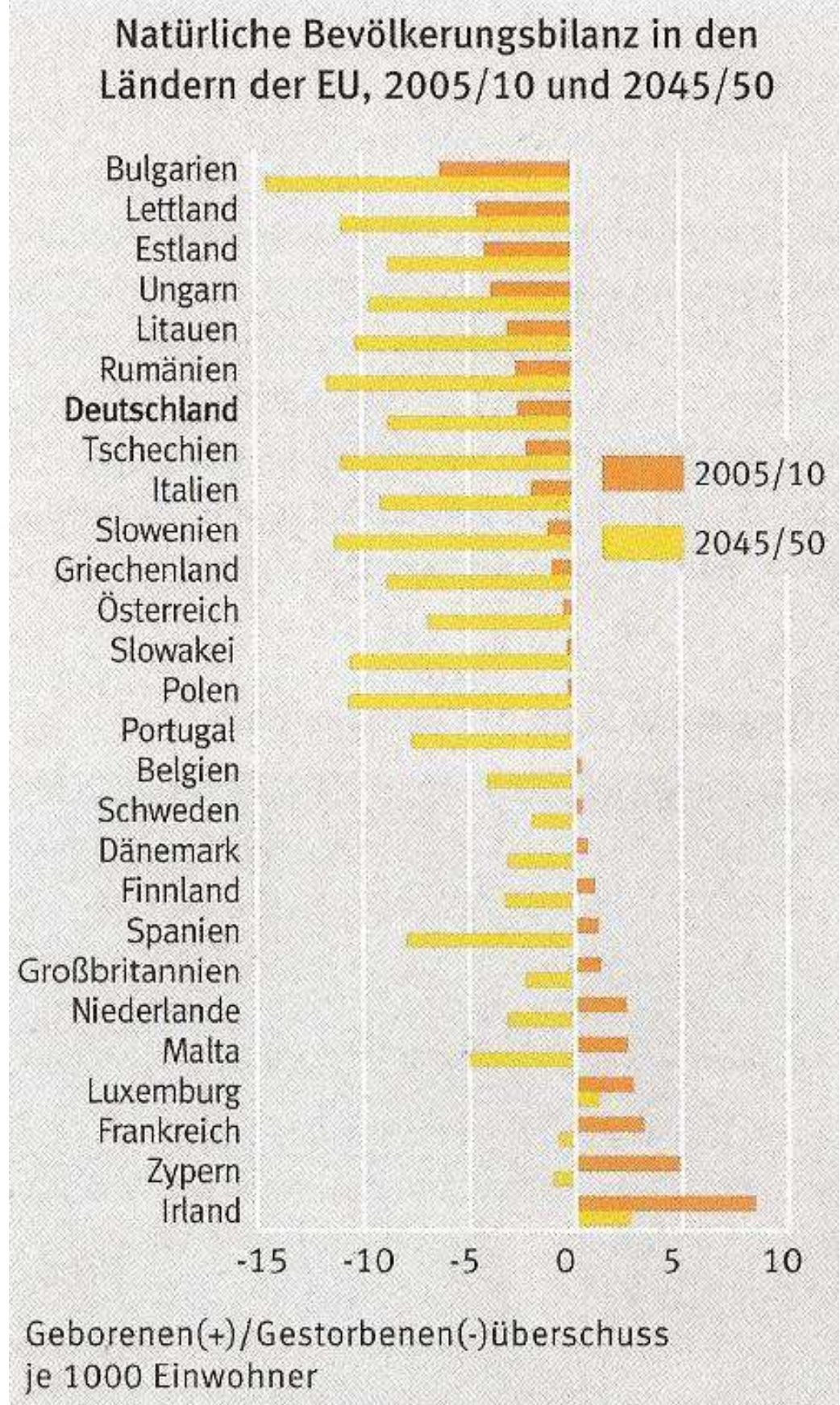

Quelle: Bundesinstitut für Bevölkerungsforschung (Hrsg.), Daten, Fakten, Trends zum demographischen Wandel in Deutschland, 2008, S. 31. 
Anlage 3:

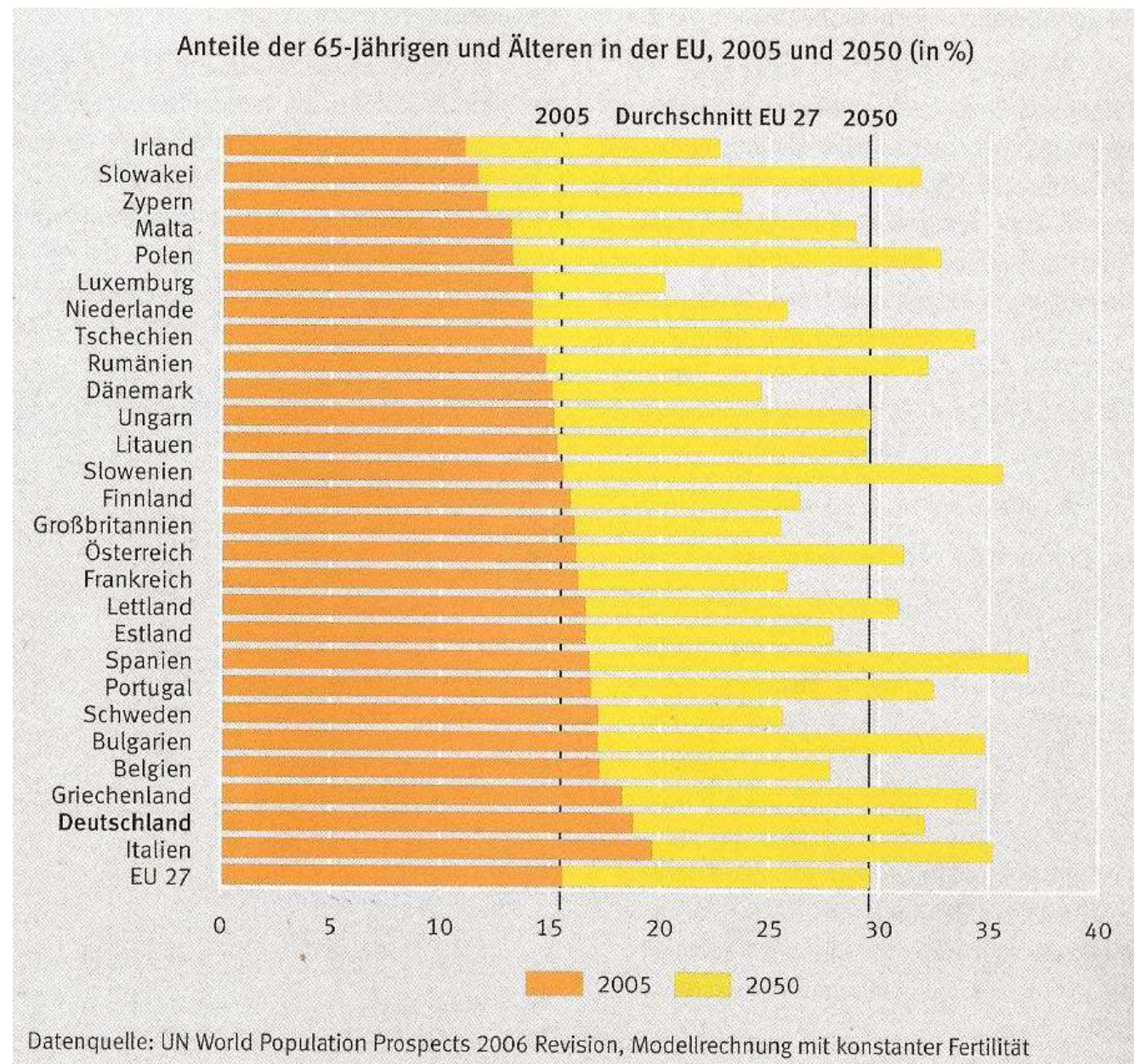

Quelle: Bundesinstitut für Bevölkerungsforschung (Hrsg.), Daten, Fakten, Trends zum demographischen Wandel in Deutschland, 2008, S. 30. 
Anlagen 4a und 4b:

EU - 27 : Erwerbstätigenquoten der 55 - 64-jährigen und der 65+ in 2009 in \%.

\section{Abb 3.6 Erwerbstätigenquoten der 55- bis 64-Jährigen 2009 in \%}

Schweden

Estland

Dänemark

Vereinigtes Königreich

\section{Deutschland}

Zypern

Finnland

Niederlande

Lettland

Litauen

irland

Portugal

Tschechische Republik Bulgarien

Spanien

Rumänien

Griechenland

Österreich

Slowakei

Frankreich

Luxemburg

Italien

Slowenien

Belgien

Ungarn

Polen

Malta

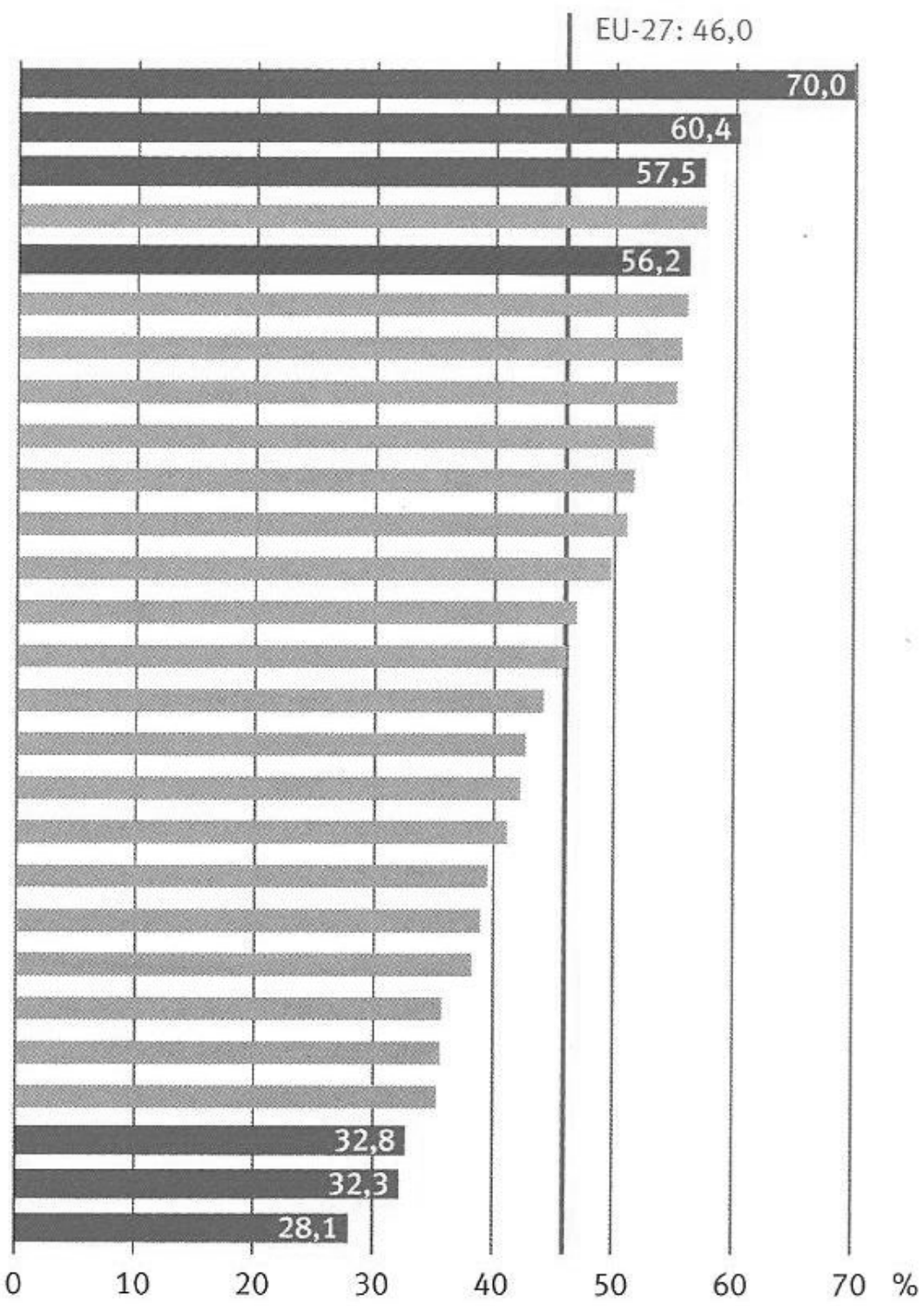




\section{Abb 3.8 Erwerbstätigenquoten 65+ 2009 in \%}

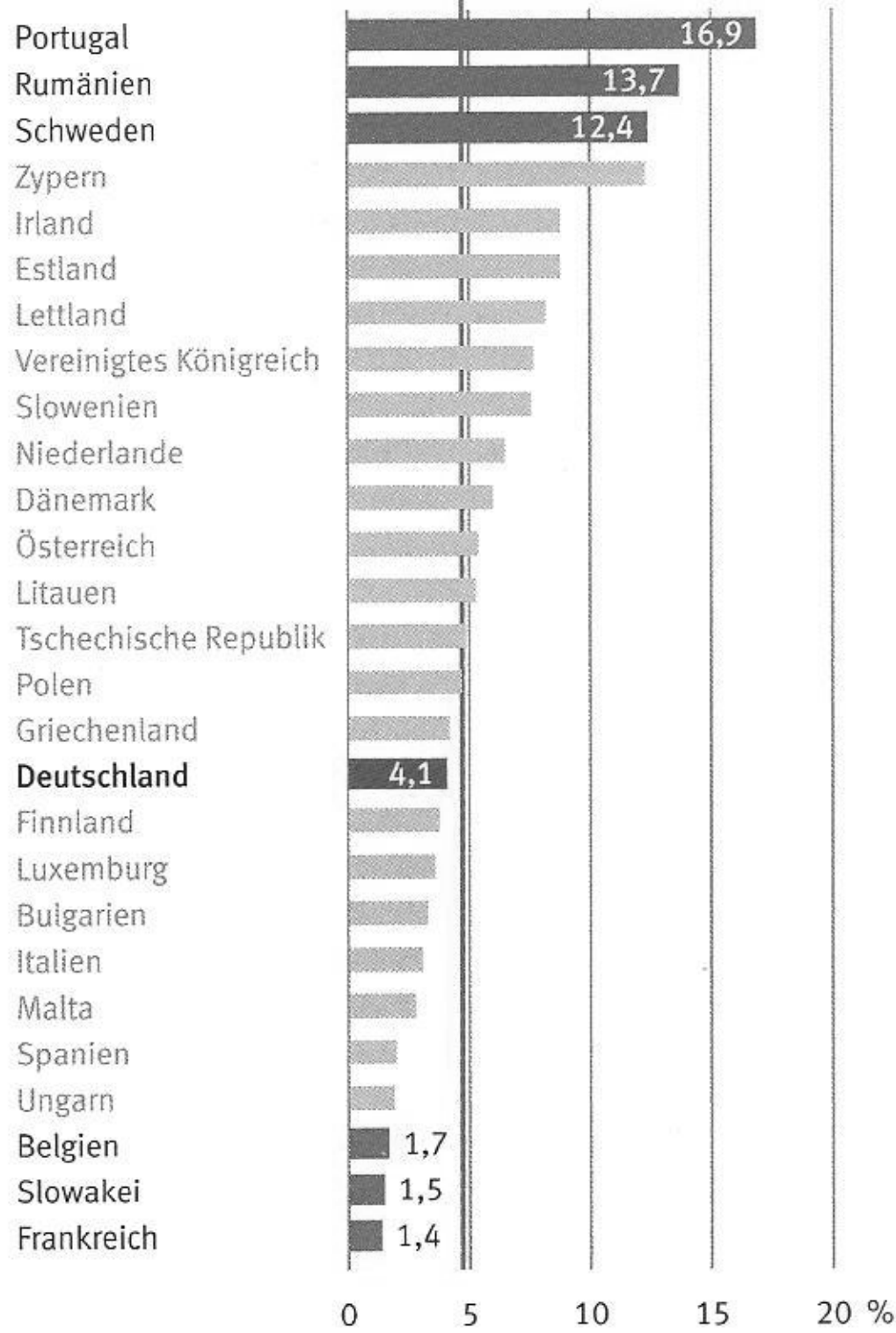

Quelle: Statistisches Bundesamt, Im Blickpunkt: Ältere Menschen in Deutschland und der EU, 2011, S. 49 und S. 51. 
Anlage 5:

Abb. 9: Erwerbstätigenquote der 20- bis 64-Jährigen 2010, in \%

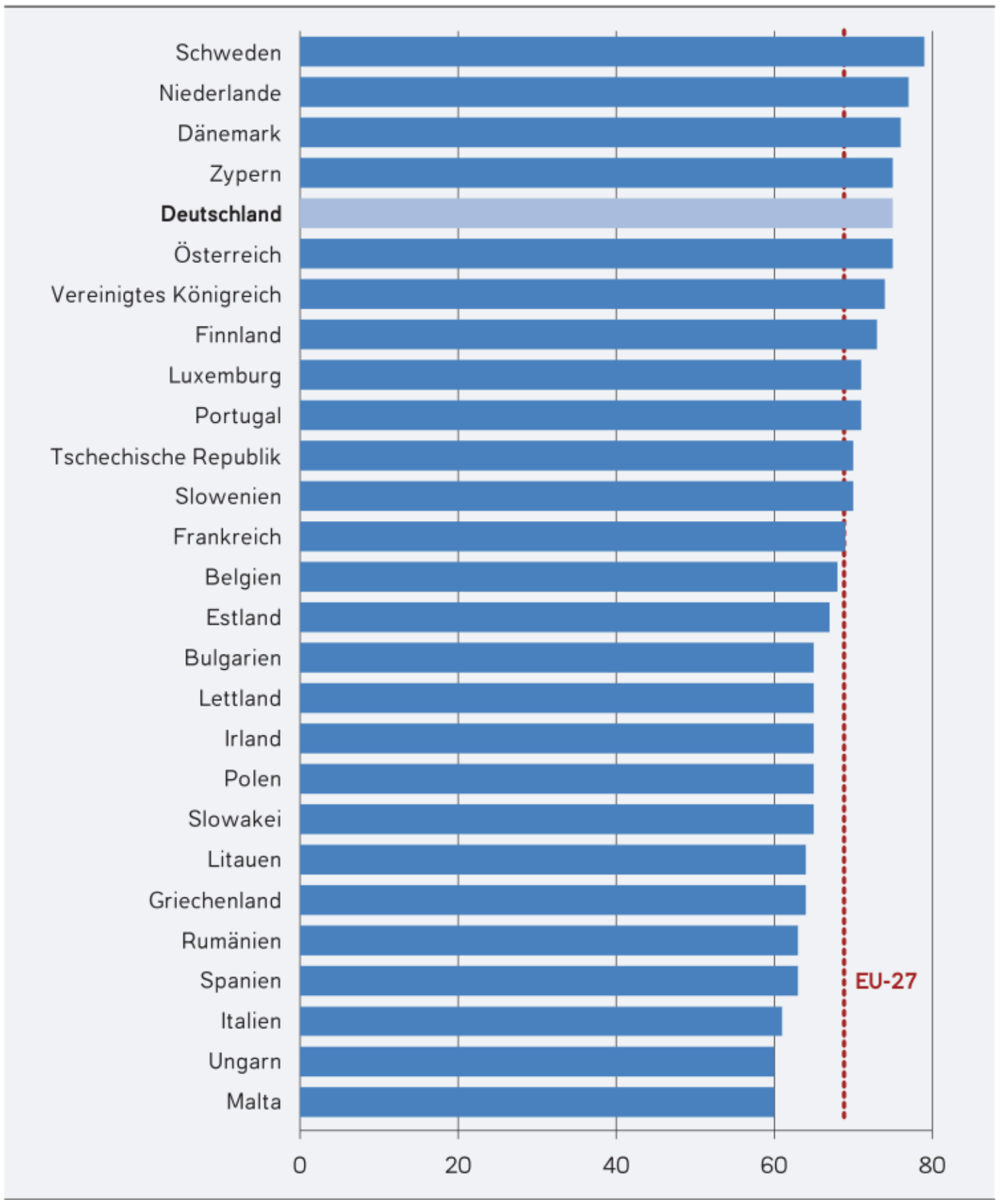

Quelle: Statistisches Bundesamt (Hrsg.), Datenreport 2011 Band II, 2011, S. 406. 
